\title{
High-Rate Redundant Space-Time Coding
}

\author{
Jawad Manssour, ${ }^{1}$ Afif Osseiran, ${ }^{1}$ and Slimane Ben Slimane ${ }^{2}$ \\ ${ }^{1}$ Ericsson AB, 16480 Stockholm, Sweden \\ ${ }^{2}$ Communication Systems, Royal Institute of Technology (KTH), 16440 Stockholm, Sweden
}

Correspondence should be addressed to Jawad Manssour, jawad.manssour@ericsson.com

Received 26 September 2009; Accepted 27 December 2009

Academic Editor: Adnan Kavak

Copyright (C) 2010 Jawad Manssour et al. This is an open access article distributed under the Creative Commons Attribution License, which permits unrestricted use, distribution, and reproduction in any medium, provided the original work is properly cited.

\begin{abstract}
We present a new space-time encoder based on packet-level redundancy which can increase the space-time encoder rate beyond unity without compromising diversity gains. A complementary low-complexity decoding algorithm based on maximum ratio combining and successive interference cancelation is further proposed. A major merit of the decoding algorithm is that it allows to adaptively tradeoff between diversity and multiplexing gains based on the estimated channel parameters at the receiver without requiring any channel state information at the transmitter. System level simulation results give insight into the advantages of the proposed scheme when compared to its Alamouti and MIMO multiplexing based on single value decomposition counterparts.
\end{abstract}

\section{Introduction}

The usage of multiple antennas has proven to be a good remedy to the unreliability of the wireless channel as it offers significant diversity and/or multiplexing gains relative to single antenna systems. In particular, space-time coding (STC) $[1,2]$ has attracted a significant amount of research due to its potential to improve the transmission's reliability. A general trend in current space-time code design is that different symbols are transmitted independently of each other. However, by performing some linear combining on the packets present at the input of the space-time encoder, the number of packets to be transmitted is effectively decreased, thereby increasing the rate of the encoder while ultimately preserving the desired redundancy.

In this work, we present a new way of generating spacetime codes which is based on combining two (or more) data symbols into one, in a way similar to network coding [3]. However, in the proposed method, the coding takes place at the transmitting node instead of at an intermediate node. Such a design can be exploited in order to generate highrate and redundant space time codes. An example of such space-time codes is presented and a complementing lowcomplexity decoding algorithm based on successive interference cancelation (SIC) and maximum ratio combining (MRC) is proposed. The decoding algorithm can exploit the redundancy introduced in order to adaptively tradeoff between diversity and multiplexing gains for the different streams without requiring any channel state information (CSI) at the transmitter. A proof of concept is presented through system level simulation results.

The rest of this paper is organized as follows. Section 2 introduces the proposed STC and explains the corresponding decoding algorithm. Section 3 contains the SINR and sumcapacity derivations. In Section 4, the simulation environment is explained. The system-level simulation results are presented in Section 5. Lastly, conclusions are given in Section 6.

\section{Redundant and High-Rate Coding}

We propose a space time block encoder where at least one of the transmit instances uses a finite field encoding operation between at least two data elements. For instance, a bit wise modulo-2 operation may be applied to the bits of different data streams. As the bits from two (or more) streams are combined together into one resultant stream, the space-time encoder rate can be considerably increased without compromising the desired redundancy. The resultant data streams are then mapped to the physical transmitting antennas. 
2.1. System Model. In this work, we limit our study to the case of a $2 \times 2$ MIMO system; however, the generalization to an $m \times n$ MIMO system is analogous. The channel matrix is given by

$$
\mathbf{H}=\left[\begin{array}{ll}
h_{11} & h_{21} \\
h_{12} & h_{22}
\end{array}\right],
$$

where $h_{i j}$ refers to the channel between transmitting antenna $i$ and receiving antenna $j$.

At the output of the encoder, the following coded matrix is assumed:

$$
\mathbf{C}^{\mathbf{T}}=\left[\begin{array}{llll}
c_{11} & c_{21} & \ldots & c_{K 1} \\
c_{12} & c_{22} & \ldots & c_{K 2}
\end{array}\right],
$$

where $K$ is the codeword length of the space time encoder and the rows of $\mathbf{C}^{\mathbf{T}}$ represent the transmitting antenna. The coded matrix contains the bitwise manipulated bits using the proposed method prior to antenna mapping and modulation. The choice of $c_{i j}$ depends on the desired spatial multiplexing and/or diversity gains based on the operating channel conditions. However, due to space restrictions, we only present one example of the proposed space-time codes in the following.

2.2. An Example of the Proposed Space-Time Codes. A $2 \times 2$ Alamouti scheme can provide a diversity order up to 4 , albeit with a rate equal to 1 . However, it would be desirable to be able to exchange some of this diversity gain into multiplexing gains under certain channel conditions. This aim can be achieved by using the following coded transmission matrix:

$$
\mathbf{C}^{\mathrm{T}}=\left[\begin{array}{ll}
s_{1} & s_{2} \oplus s_{3} \\
s_{2} & s_{1} \oplus s_{3}
\end{array}\right]=\left[\begin{array}{ll}
s_{1} & x_{1} \\
s_{2} & x_{2}
\end{array}\right],
$$

where $x_{1}=s_{2} \oplus s_{3}$, and $x_{2}=s_{1} \oplus s_{3}$. Note that $x_{i}$ has been introduced to simplify the notation and that the $\oplus$ operation represents a modulo-2 addition on the binary bits of the symbols $s_{i}$ and $x_{i}$. With the modulo- 2 addition operating on binary bits of the symbols, any type of modulation can be used on the transmitted codewords (e.g., based on channel conditions). It can be seen that the codewords consist of two time slots where three different symbols are transmitted thus providing a transmission rate of $3 / 2$. The main advantage of the proposed scheme is that each of the three symbols has been transmitted twice, each on the two different antennas, a fact that will be exploited by the decoding algorithm to achieve the desired diversity-multiplexing tradeoff.

\section{SINR and Sum-Capacity Derivation}

In the following we will derive the signal-to-interferenceand-noise-ratio (SINR) equations based on MRC and SIC at the receiver. (Alternatively, Minimum Mean Square Error (MMSE) or Maximum Likelihood (ML) decoding may as well be used.) The transmission protocol consists of two transmission slots, $T_{1}$ and $T_{2}$, during which the channel remains constant. Both antennas transmit with equal power $p$.
3.1. Predecoding SINR. The predecoding SINR is the SINR computed based on the signals received after transmissions during the times $T_{1}$ and $T_{2}$, prior to symbol decoding and combining. The received signals at the first and second antennas are given by ( in the algorithm flowchart in Figure $1, y_{j}\left(T_{k}\right)$ is represented as $\left.y_{j k}\right)$

$$
\mathbf{Y}=\left[\begin{array}{ll}
y_{1}\left(T_{1}\right) & y_{1}\left(T_{2}\right) \\
y_{2}\left(T_{1}\right) & y_{2}\left(T_{2}\right)
\end{array}\right]=\mathbf{H C}^{\mathrm{T}}+\boldsymbol{\Xi},
$$

where $\mathbf{H}$ is the channel matrix defined in (1), $y_{j}\left(T_{k}\right)$ is the received signal at the $j$ th receive antenna for the transmission phase $T_{k}, \mathbf{C}^{\mathrm{T}}$ is the coded matrix in (3), and $\boldsymbol{\Xi}$ is the instantaneous noise and interference given by

$$
\boldsymbol{\Xi}=\left[\begin{array}{ll}
\xi_{1}\left(T_{1}\right) & \xi_{1}\left(T_{2}\right) \\
\xi_{2}\left(T_{1}\right) & \xi_{2}\left(T_{2}\right)
\end{array}\right],
$$

where $\xi_{j}\left(T_{k}\right)$ is the noise and interference at the $j$ th receive antenna for the transmission phase $T_{k}$.

The baseband received symbol at the $j$ th receive antenna for transmission phase $T_{k}$ is given by

$$
y_{j}\left(T_{k}\right)=h_{1, j}\left(T_{k}\right) c_{k 1}+h_{2, j}\left(T_{k}\right) c_{k 2}+\xi_{j}\left(T_{k}\right)
$$

where $c_{k 1}$ and $c_{k 2}$ are the transmitted symbols during $T_{k}$ from antennas 1 and 2, respectively.

Depending on the received signal strength, we can distinguish between two cases.

3.1.1. Case 1. In the first case, the received power from the first transmit antenna is stronger than the received power from the second transmit antenna. In that case $s_{1}$ (resp., $x_{1}$ ) is detected first during $T_{1}$ (resp., $T_{2}$ ) by combining through, for example, MRC the streams from the two receive antennas and treating $s_{2}$ (resp., $x_{2}$ ) as interference, then followed by detecting $s_{2}$ (resp., $x_{2}$ ) after performing SIC of the already decoded $s_{1}$ (resp., $x_{1}$ ). The predecoding SINRs are then given by

$$
\begin{aligned}
& \Gamma_{s_{1}}=\Gamma_{x_{1}}=\frac{p\left|h_{11}\right|^{2}}{p\left|h_{21}\right|^{2}+2 \sigma_{1}^{2}}+\frac{p\left|h_{12}\right|^{2}}{p\left|h_{22}\right|^{2}+2 \sigma_{2}^{2}}, \\
& \Gamma_{x_{2}}=\Gamma_{s_{2}}=\frac{p\left|h_{21}\right|^{2}}{2 \sigma_{1}^{2}}+\frac{p\left|h_{22}\right|^{2}}{2 \sigma_{2}^{2}},
\end{aligned}
$$

where $2 \sigma_{i}^{2}=E\left\{\left|\xi_{i}\left(T_{k}\right)\right|^{2}\right\}$ is the average interference plus noise power at the $i$ th receive antenna which is assumed constant over the two time slots.

3.1.2. Case 2. In the second case, the received power from the second transmit antenna is stronger than the received power from the first transmit antenna. Here $s_{2}$ and $x_{2}$ are first detected in their respective time slots, followed by $x_{1}$ and 


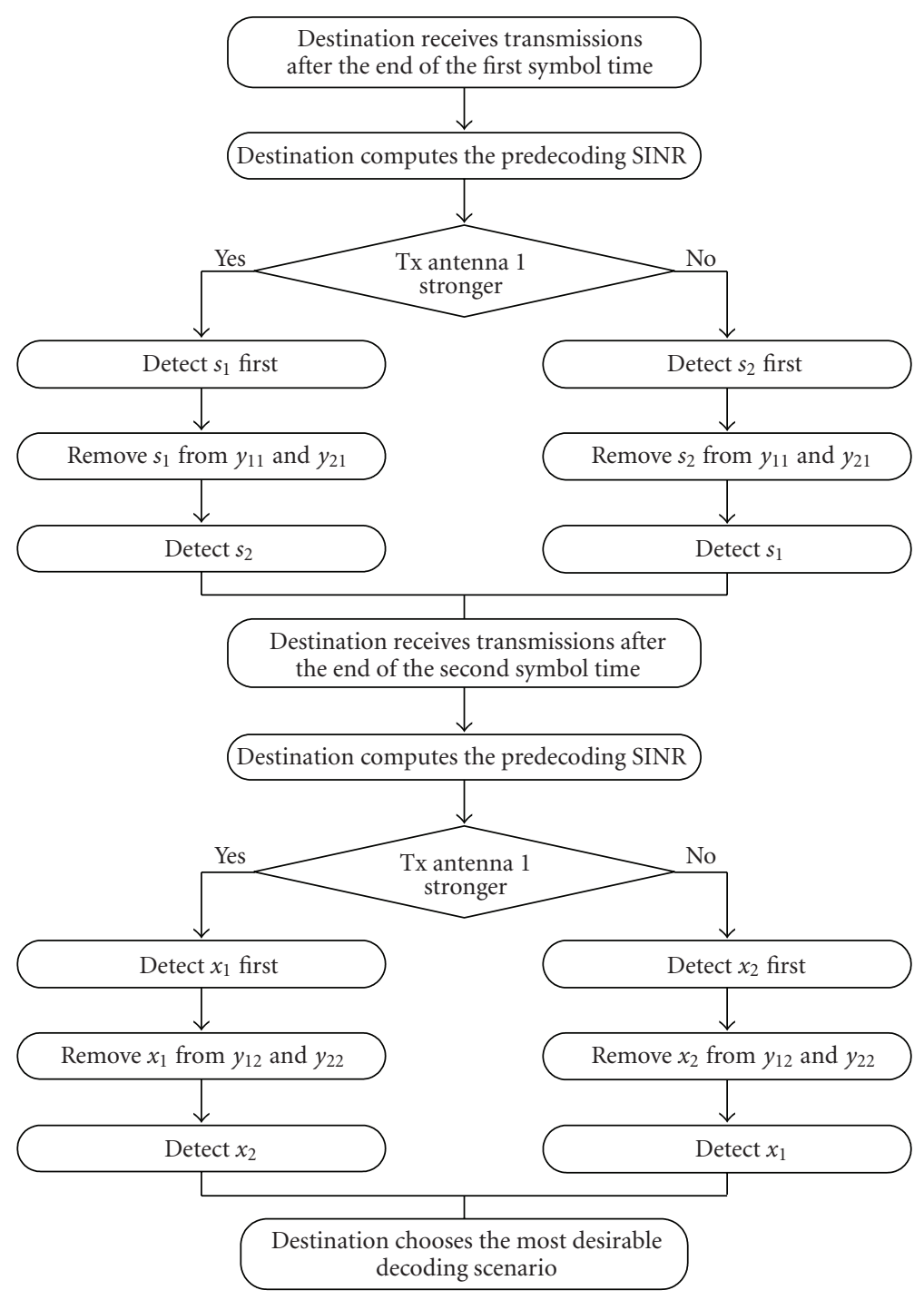

FIGURE 1: Flowchart of the proposed decoding algorithm.

$s_{1}$. Similarly to Case 1 , assuming that $s_{2}$ and $x_{2}$ are correctly decoded, the predecoding SINRs can be obtained as

$$
\begin{aligned}
& \Gamma_{s_{2}}=\Gamma_{x_{2}}=\frac{p\left|h_{21}\right|^{2}}{p\left|h_{11}\right|^{2}+2 \sigma_{1}^{2}}+\frac{p\left|h_{22}\right|^{2}}{p\left|h_{12}\right|^{2}+2 \sigma_{2}^{2}}, \\
& \Gamma_{x_{1}}=\Gamma_{s_{1}}=\frac{p\left|h_{11}\right|^{2}}{2 \sigma_{1}^{2}}+\frac{p\left|h_{12}\right|^{2}}{2 \sigma_{2}^{2}} .
\end{aligned}
$$

3.2. Postdecoding SINR. Following the SINR evaluation of the transmitted coded symbols (i.e., predecoding SINR), the modulated symbols will be estimated by the spacetime decoder. Figure 1 shows a flowchart of the decoding algorithm. Once the symbols $s_{1}, s_{2}, x_{1}$, and $x_{2}$ have been detected, the decoding will be done depending on the predecoding SINR values of those symbols, resulting in the postdecoding SINRs that would directly determine the resulting capacity.
We distinguish between two main decoding scenarios that offer different diversity-multiplexing tradeoffs.

3.2.1. Scenario 1. In the first scenario, the direct links are the strongest and both $s_{1}$ and $s_{2}$ are decoded based on their direct transmissions. Consequently we use $x_{1}$ and $x_{2}$ to obtain $s_{3}$. (This is simply achieved by first decoding $s_{1} \oplus s_{3}$ with $s_{1}$ (i.e., $\left.s_{1} \oplus\left(s_{1} \oplus s_{3}\right)\right)$ and obtaining the first estimate of $s_{3}$. The second estimate of $s_{3}$ is obtained by decoding $s_{2} \oplus s_{3}$ with $s_{2}$. The two estimates of $s_{3}$ are then combined together to obtain the final decision.) In this scenario, the equivalent (i.e., postdecoding) SINRs, decoded $\Gamma^{\prime}$, for the different symbols will then be given as follows:

$$
\Gamma_{s_{1}}^{\prime}=\Gamma_{s_{1}}, \quad \Gamma_{s_{2}}^{\prime}=\Gamma_{s_{2}}, \quad \Gamma_{s_{3}}^{\prime}=\Gamma_{x_{1}}+\Gamma_{x_{2}} .
$$

3.2.2. Scenario 2. In the second scenario, one of the symbols $s_{1}$ and $s_{2}$ (the one with the higher predecoding SINR) is decoded based on its direct transmission. Consequently we 
use the relevant redundant symbol $x$ (i.e., $x_{1}$ or $x_{2}$ ) to obtain the other $s_{i}$ (with the lower predecoding SINR), and then use the other redundant $x$ to obtain $s_{3}$. The two possible cases are as follows.

Case $1\left(\Gamma_{s_{1}}>\Gamma_{s_{2}}\right)$. In this case, the symbol $s_{1}$ is decoded directly, the redundant symbol $x_{1}$ is used to increase the diversity gain of the symbol $s_{2}$, and the redundant symbol $x_{2}$ is used to obtain a multiplexing gain by decoding $s_{3}$. The equivalent postdecoding SINRs of the different transmitted symbols will then be given by

$$
\Gamma_{s_{1}}^{\prime}=\Gamma_{s_{1}}, \quad \Gamma_{s_{3}}^{\prime}=\Gamma_{x_{2}}, \quad \Gamma_{s_{2}}^{\prime}=\Gamma_{s_{2}}+\Gamma_{x_{1}} .
$$

Case $2\left(\Gamma_{s_{2}}>\Gamma_{s_{1}}\right)$. In this case, the symbol $s_{2}$ is decoded directly, the redundant symbol $x_{2}$ is used to increase the diversity gain of the symbol $s_{1}$, and the redundant symbol $x_{1}$ is used to obtain multiplexing gain by decoding $s_{3}$.

The equivalent SINRs of the different transmitted symbols will then be given by

$$
\Gamma_{s_{2}}^{\prime}=\Gamma_{s_{2}}, \quad \Gamma_{s_{3}}^{\prime}=\Gamma_{x_{1}}, \quad \Gamma_{s_{1}}^{\prime}=\Gamma_{s_{1}}+\Gamma_{x_{2}} .
$$

3.3. Capacity Calculation. Using the postdecoding SINRs derived in the previous subsection, the sum-capacity for all the different scenarios can be written as follows:

$$
C_{\text {sum }}=\sum_{i=1}^{3} \log _{2}\left(1+\Gamma_{s_{i}}^{\prime}\right),
$$

where the postdecoding SINRs depend on the chosen decoding scenario. A main merit of the proposed scheme is that the diversity-multiplexing gains for the different transmitted streams can be adaptively controlled at the receiver based on the estimated channel coefficients in conjunction with perstream desired performance measures by simply choosing the desirable, yet feasible decoding scenario (i.e., if the channel coefficients permit), consequently not requiring any CSI at the transmitter. Furthermore, it makes it possible to employ low-complexity decoding.

\section{Simulation Environment}

A network deployment with seven cells is considered in order to measure the performance in the presence of inter-cell interference. Each cell has a radius of $500 \mathrm{~m}$, and a reuse factor of 1 is assumed. All transmitting and receiving nodes are assumed to have two uncorrelated antennas each. The C2 metropolitan area pathloss and channel model from [4] are used in the evaluations. Non-line-of-sight propagation is assumed between the BS antennas and the transmitters. Shadow fading is log-normally distributed with a standard deviation of $8 \mathrm{~dB}$. The capacity evaluation is based on the Shannon model.

As the main goal of this work is to evaluate the capacity performance of the proposed scheme, we opt to use systemlevel simulation. The evaluation of the error performance, hence the usage of link-level simulation, is left as a future work. However, the SINR results that were included should give a hint about the robustness of each simulated scheme.

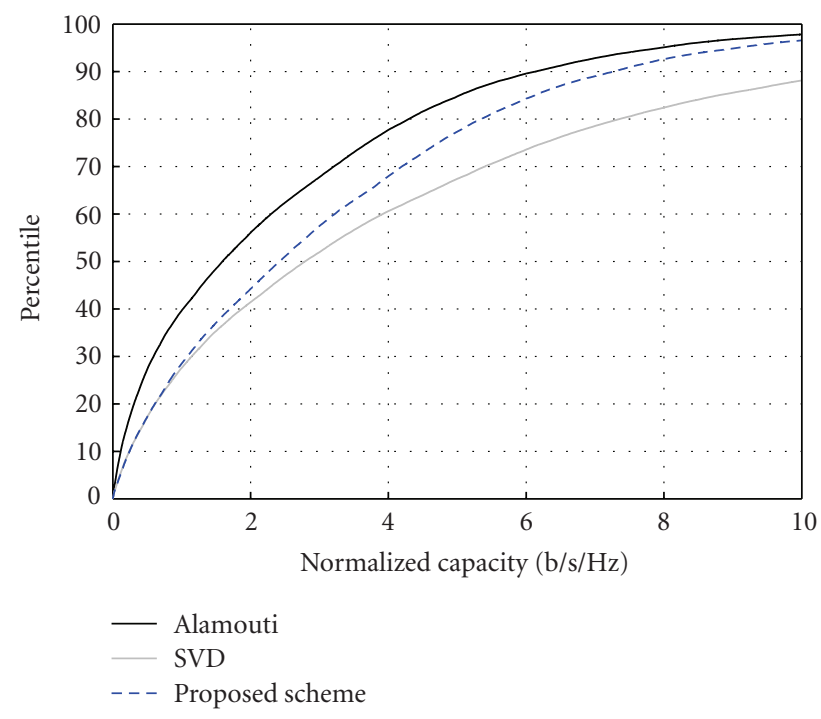

Figure 2: Normalized capacity of the evaluated schemes.

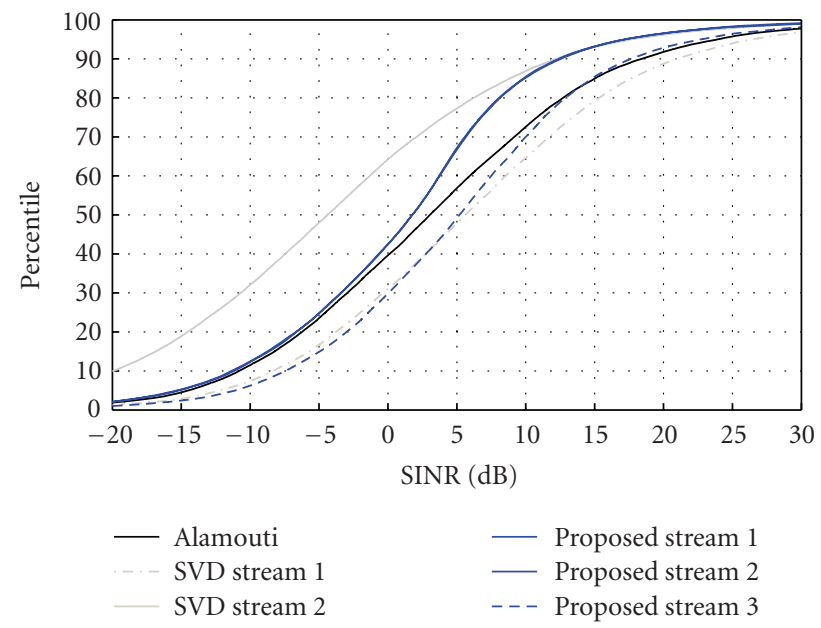

FIGURE 3: SINR performance of the evaluated schemes.

\section{System-Level Performance Results}

The proposed scheme is evaluated and compared to the $2 \times 2$ Alamouti scheme with MRC combining at the receiver and the $2 \times 2$ channel capacity based on single value decomposition (SVD). The cumulative distribution function $(\mathrm{CDF})$ of the sum-capacity is shown in Figure 2. The average normalized sum-capacity of the Alamouti scheme is $2.49[\mathrm{~b} / \mathrm{s} / \mathrm{Hz}]$, whereas that of the SVD method is $4.42[\mathrm{~b} / \mathrm{s} / \mathrm{Hz}]$, and the proposed scheme is $3.52[\mathrm{~b} / \mathrm{s} / \mathrm{Hz}]$. Furthermore, the CDF of the SINR performance is shown in Figure 3 where different streams of a same method have a similar performance during $T_{1}$ and $T_{2}$ due to the block fading assumption. Although the SVD method achieves the highest sum-capacity, it has two major drawbacks: it requires full CSI at both the transmitter and the receiver, and half of the transmitted streams will have a very low performance (which might not yield any gains in a practical setup). On the 
other hand, the proposed method is able to exchange one of the transmitted streams into a better diversity performance so that the diversity-multiplexing behavior can be controlled by the receiver as opposed to the Alamouti scheme that provides a better diversity performance in general at the expense of a lower rate. Another main merit of the proposed scheme over the Alamouti method is that whereas the latter fails in case of non block fading, this setup would provide more diversity gains to the former.

\section{Conclusion}

In this work, we suggested the imitation of simple linear network coding at transmitters possessing multiple antennas, and we showed how this could be exploited in order to design redundant high-rate space-time codes. We proposed a complementary low-complexity decoding algorithm based on successive interference cancelation that can adaptively tradeoff between diversity and multiplexing gains without requiring any channel state information at the transmitting side. System level simulation results were presented as a proof of concept and to gain insight into the advantages of the proposed scheme.

\section{References}

[1] V. Tarokh, N. Seshadri, and A. R. Calderbank, "Space-time codes for high data rate wireless communication: performance criterion and code construction," IEEE Transactions on Information Theory, vol. 44, no. 2, pp. 744-765, 1998.

[2] S. M. Alamouti, "A simple transmit diversity technique for wireless communications," IEEE Journal on Selected Areas in Communications, vol. 16, no. 8, pp. 1451-1458, 1998.

[3] R. Ahlswede, N. Cai, S.-Y. R. Li, and R. W. Yeung, "Network information flow," IEEE Transactions on Information Theory, vol. 46, no. 4, pp. 1204-1216, 2000.

[4] E. J. Meinilä, "Final report on link level and system level channel models,” Tech. Rep. IST-2003-507581 WINNER I, D5.4 v. 1.4, 2005, http://projects.celtic-initiative.org/winner+. 

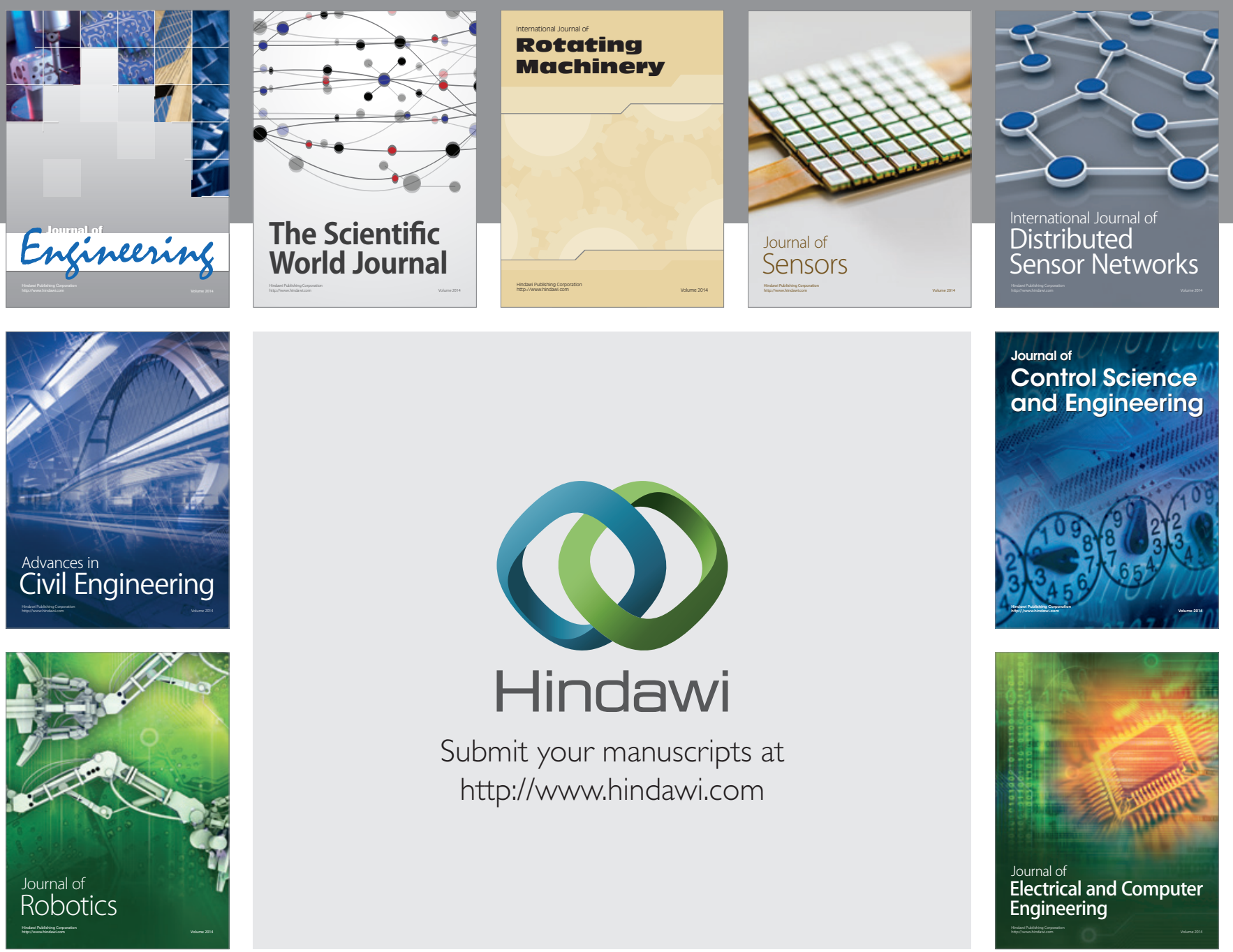

Submit your manuscripts at

http://www.hindawi.com
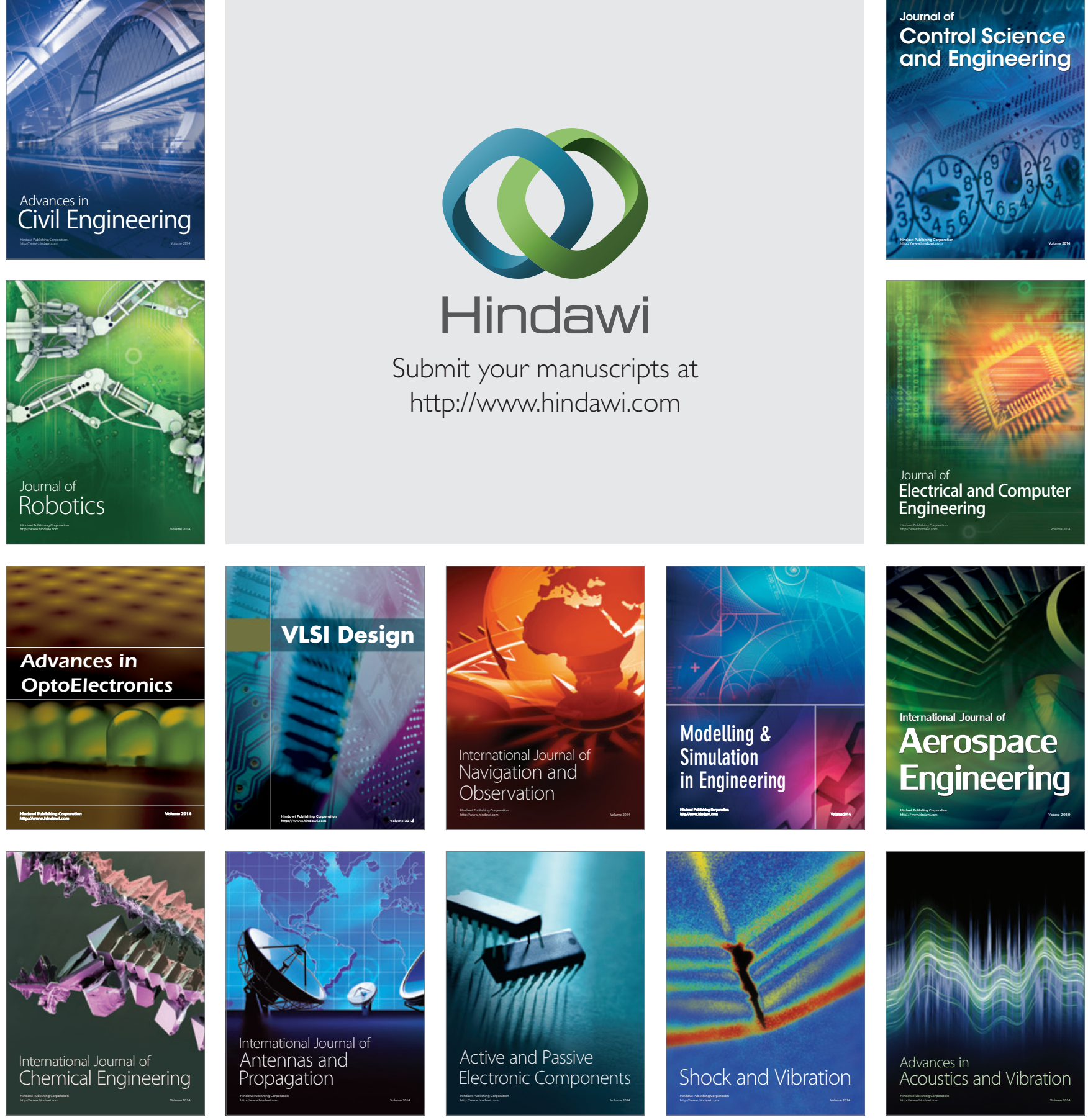